\title{
Lactic Acidosis and Hypoglycemia in a Patient with Gastric Diffuse Large B-Cell Lymphoma due to the Warburg Effect
}

\author{
Takafumi Hamada ${ }^{a}$ Toshinari Kaku ${ }^{a}$ Sumitaka Mitsu ${ }^{a} \quad$ Yoshinori Morita ${ }^{a}$ \\ Nobuhito Ohno ${ }^{b}$ Hironori Yamaguchic \\ aDepartment of General Internal Medicine, Kagoshima Prefectural Oshima Hospital, \\ Kagoshima, Japan; 'bepartment of Hematology, Ikeda Hospital, Kagoshima, Japan; \\ 'Department of Medical Oncology, Jichi Medical University Hospital, Tochigi, Japan
}

\section{Keywords}

Diffuse large B-cell lymphoma · Lactic acidosis · Hypoglycemia · Warburg effect

\begin{abstract}
Lactic acidosis is pathophysiologically classified into type A and type B. The latter is a rare but potentially life-threatening emergency, mainly described in hematological malignancies. The association between Type B lactic acidosis and malignancy is known as the Warburg effect. Patients with the Warburg effect have a very poor prognosis. Herein, we report a case of gastric diffuse large $\mathrm{B}$-cell lymphoma ( $\mathrm{DLBCL}$ ) with severe lactic acidosis and hypoglycemia owing to the Warburg effect that were effectively treated by prompt introduction of chemotherapy. A 73-year-old woman with a 2-month history of abdominal distension was referred to us for suspected peritoneal cancer. Pathological examination revealed gastric DLBCL with peritoneal dissemination. After hospitalization, blood test results revealed prolonged hypoglycemia, with a blood sugar level of $50-70 \mathrm{mg} / \mathrm{dL}$; severe lactic acidosis with $\mathrm{pH} 7.166$; lactate level $12.7 \mathrm{mmol} / \mathrm{L}$; and base excess $-21.0 \mathrm{mEq} / \mathrm{L}$, despite continuous administration of glucose and sodium bicarbonate. The cause of lactic acidosis and/or hypoglycemia was considered to be the Warburg effect. We initiated a 50\% reduced-dose CHOP (cyclophosphamide, vincristine, doxorubicin, prednisolone) chemotherapy regimen without rituximab until information on the CD20-positive status was available. During chemotherapy, acidosis, hypoglycemia, and impaired consciousness promptly improved. If lactic acidosis or hypoglycemia is present in patients with malignant tumors, it is important to suspect the possibility of the Warburg effect and to introduce cancer treatment as soon as possible.




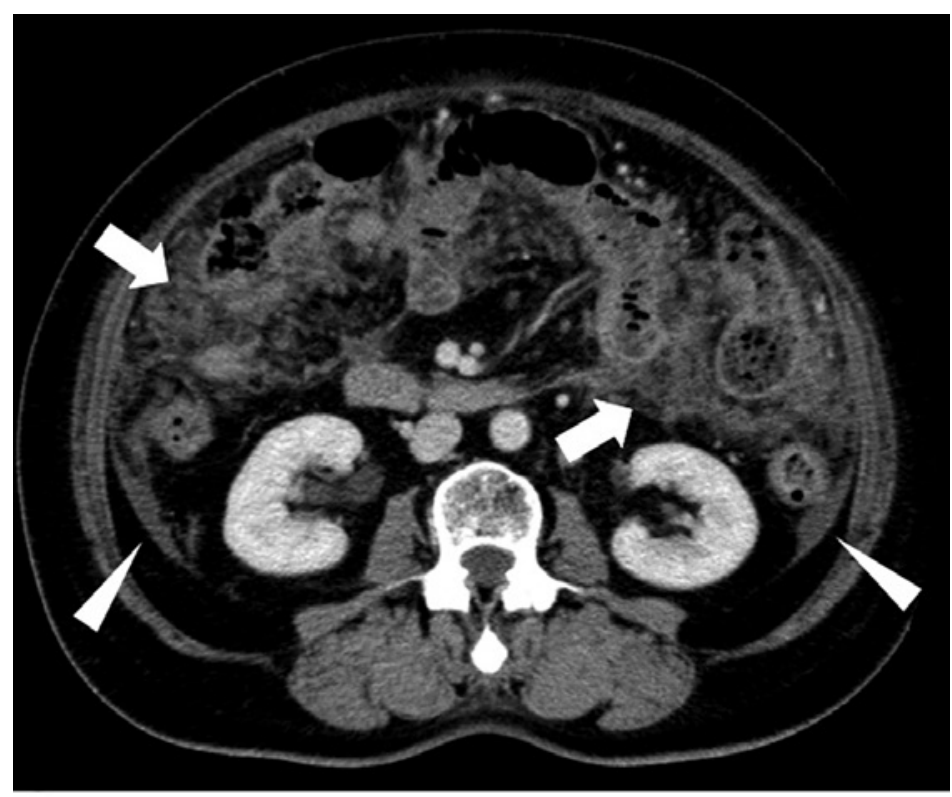

Fig. 1. Pretreatment CT images show peritoneal thickening (arrows) and a small amount of ascites (arrowheads).

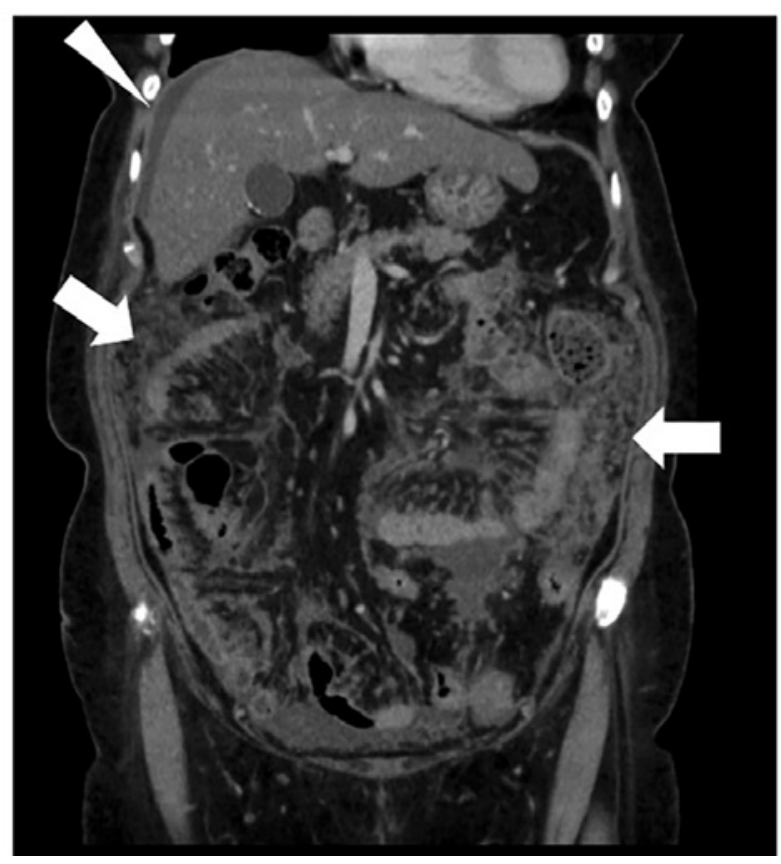

\section{Introduction}

The association between type B lactic acidosis and malignancy is known as the Warburg effect $[1,2]$. The Warburg effect is a pathological condition in which cancer cells metabolize glucose to lactic acid under aerobic conditions and produce energy dependent on glycolysis, resulting in combined lactic acidosis and hypoglycemia [1,2]. Malignant tumors are rarely associated with lactic acidosis, which is rapidly progressive and fatal if not promptly recognized and treated [3]. The majority of patients with cancer who present with cancer-related elevated lactate levels are adults with rapidly progressive leukemia or lymphoma [4]. There are only few reported cases of lactic acidosis as a symptom of diffuse large B-cell lymphoma (DLBCL). 


\section{Case Reports in Oncology}

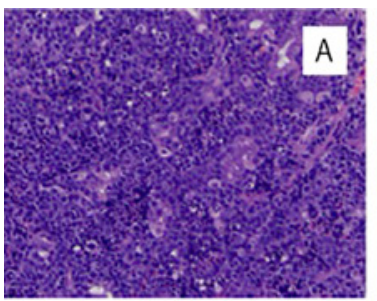

$H \& E$
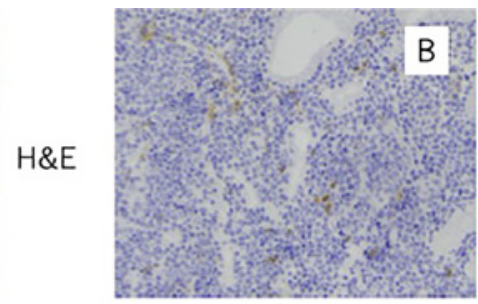

$\mathrm{CD} 10$
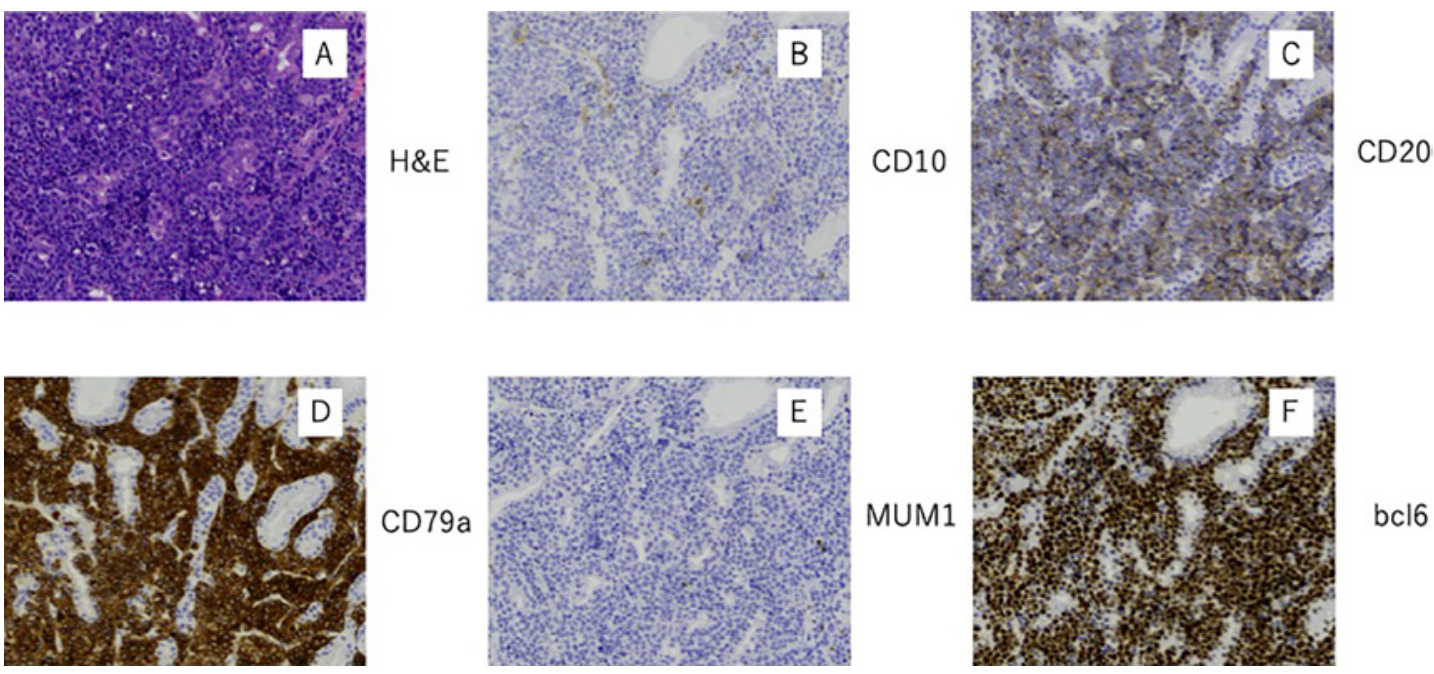

CD79a

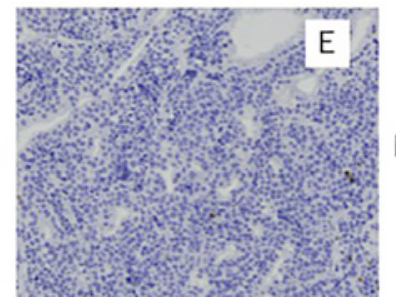

MUM1

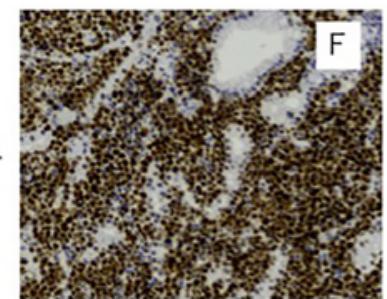

Fig. 2. A H\&E staining of the biopsy specimens showed atypical large lymphocytic infiltrates with an irregular nucleus. B-F Immunohistochemical studies showed that the atypical large cells were positive for CD20, CD79a, and BCL-6, and negative for CD3, CD10, and MUM1. Original magnification, $\times 40$.

Herein, we describe a case of DLBCL with severe lactic acidosis and hypoglycemia owing to the Warburg effect that were effectively treated by prompt introduction of chemotherapy.

\section{Case Report/Case Presentation}

A 73-year-old woman with a history of hypothyroidism and hypertension visited another hospital because of a several-day history of nausea and vomiting and abdominal distension for several months. She had no family history of malignancy or neurological disease.

Arterial blood gas analysis showed lactic acidosis with increased anion gap (pH 7.248, base excess $-10.6 \mathrm{mEq} / \mathrm{L}$, lactate level $7.30 \mathrm{mmol} / \mathrm{L}$ ). Her blood glucose level was $78 \mathrm{mg} / \mathrm{dL}$. Abdominal CT imaging revealed peritoneal thickening and a small amount of ascites (Fig. 1).

She was referred to us for suspected peritoneal cancer or peritoneal dissemination of cancer of unknown primary site. On admission, she was afebrile, with a heart rate of 100 beats/min, blood pressure of 173/90 $\mathrm{mm} \mathrm{Hg}$, respiratory rate of 20 breaths/min, and oxygen saturation at $98 \%$ on room air.

On physical examination, cardiac and pulmonary auscultation revealed normal findings. There was mild tenderness on abdominal examination, but no splenomegaly. The superficial lymph nodes were not palpable. After admission, esophagogastroduodenoscopy revealed a submucosal tumor-like mass with ulceration localized in the anterior wall of the gastric antrum and the greater curvature, and a few small flat elevated lesions with an apical depression in the gastric body. Biopsy was performed at the periphery of the ulcer and small flat elevated lesions. Histologically, the H\&E sections of the biopsy specimens showed atypical large lymphocytic infiltrates with an irregular nucleus. Immunohistochemical studies showed that the atypical large cells were positive for CD20, CD79a, and BCL-6 and negative for CD3, CD10, and MUM1 (Fig. 2); therefore, we diagnosed the patient as having DLBCL.

Exploratory laparoscopy revealed disseminated nodules in the inferior abdominal omentum, mesenteric fatty tissue, and intestinal serosa, as well as pale bloody ascites. A disseminated nodule from the omentum was excised and submitted for pathological exami- 


\section{Case Reports in Oncology}

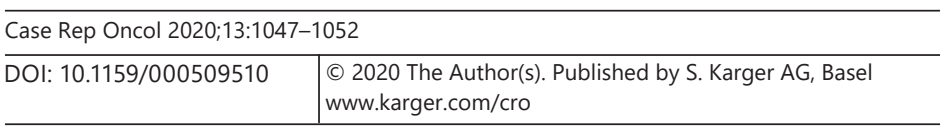

Hamada et al.: Lactic Acidosis and Hypoglycemia in Gastric DLBCL due to Warburg Effect

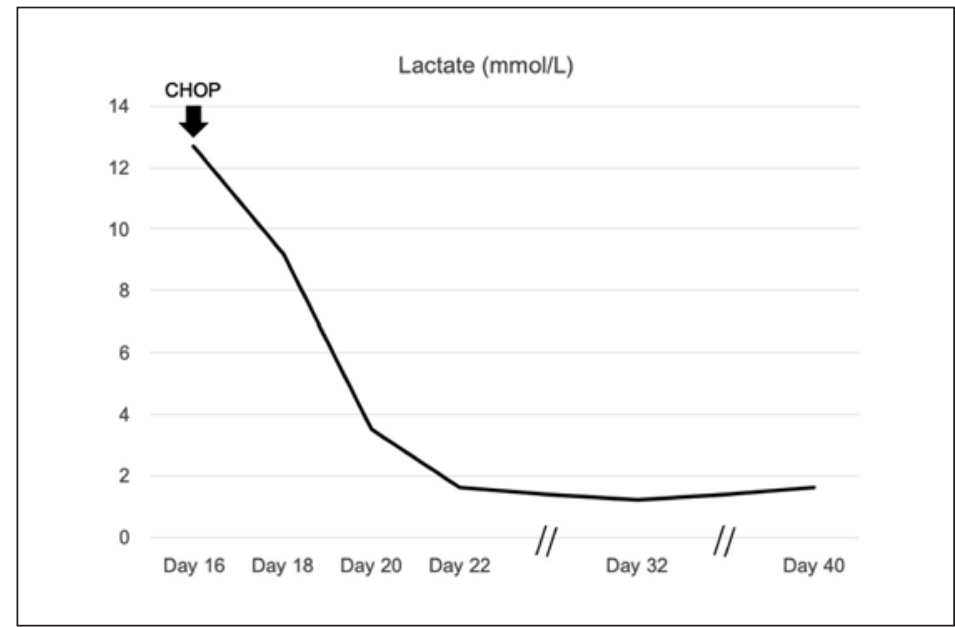

Fig. 3. A line graph showing the return of serum lactate levels to normal after the start of chemotherapy.

nation. The pathological results showed the same findings as did gastric biopsy, and the patient was diagnosed as having gastric DLBCL with peritoneal dissemination.

Blood test results after admission showed hypoglycemia with a blood glucose level of 50-70 mg/dL; lactic acidosis with pH 7.166; lactic acid $12.7 \mathrm{mmol} / \mathrm{L}$; and base excess -21.0 $\mathrm{mEq} / \mathrm{L}$. Despite continuous intravenous glucose and sodium bicarbonate administration, the hypoglycemia and lactic acidosis aggravated. The patient's consciousness was clear at the time of admission, but gradually deteriorated with respiratory failure.

At that time, we considered the Warburg effect as the cause of lactic acidosis and hypoglycemia associated with malignant lymphoma. We considered that the prompt introduction of treatment for gastric DLBCL was necessary because the Warburg effect has a poor prognosis $[5,6]$. Thus, on day 16 since admission, CHOP chemotherapy (cyclophosphamide, doxorubicin hydrochloride, vincristine, prednisolone) without rituximab was initiated with a $50 \%$ dose reduction in light of the patient's poor condition. After the treatment start, the acidosis, hypoglycemia, and impaired consciousness improved promptly.

Lactic acidosis resolved on day 22 from admission (Fig. 3). Thereafter, both the lactic acid and blood glucose levels remained within normal ranges.

On day 30 , the patient developed weakness of the bilateral lower and upper extremities. She was diagnosed with Guillain-Barré syndrome and received intravenous immunoglobulin and steroid pulse treatment. The symptoms improved with the treatment and rehabilitation.

After the first course of chemotherapy, immunohistochemical examination revealed CD20 positivity; hence, a second course of R-CHOP (rituximab, cyclophosphamide, doxorubicin hydrochloride, vincristine, prednisolone) chemotherapy at $100 \%$ dose was initiated on day 39 . On day 46, CT showed that the peritoneal thickening and ascites had almost disappeared. By day 107, the patient underwent five courses of chemotherapy. Although a total of eight courses were planned, subsequent chemotherapy was postponed as the patient developed pneumocystis pneumonia, cytomegalovirus infection, and candidemia after the fifth course. At day 226, the patient remains hospitalized and will resume chemotherapy after the infection is under control.

\section{Discussion/Conclusion}

In the present case, the patient had severe lactic acidosis as one of the early symptoms of DLBCL. Initially, peritoneal cancer was suspected at the time of admission, but histological diagnosis confirmed DLBCL. Until the histological diagnosis was made, we could not identify 
the cause of lactic acidosis. We considered that the cause was the Warburg effect and introduced chemotherapy early, which successfully improved the lactic acidosis.

Lactic acidosis occurs when there is an imbalance between production and clearance of lactate [7]. It is classified into type A and type B on the basis of the pathophysiology [7-9]. Type A lactic acidosis is the most common clinical type, reflecting poor oxygenation of tissues caused by hypoperfusion. The causes of Type A lactic acidosis include sepsis, cardiogenic shock, mesenteric ischemia, and severe dehydration [7-9]. Type B lactic acidosis occurs under physiological conditions and tissues remain normally perfused. The causes of type $B$ lactic acidosis include liver disease, vitamin $\mathrm{B}_{1}$ deficiency, alcoholism, metformin, and malignancy [7-9]. Type B lactic acidosis is a rare but potentially life-threatening emergency, mainly described in hematological malignancies, but several cases involving solid tumors have been reported as well $[9,10]$.

The association between type B lactic acidosis and malignancy is known as the Warburg effect $[1,2]$. Warburg hypothesized that under aerobic conditions, tumor tissue metabolizes more glucose to lactose than does normal tissue. Several other hypotheses have been proposed for the mechanism of the Warburg effect, but they are not clearly understood [11]. Although the Warburg effect was thought to be the consequence of carcinogenesis, it has also been proposed that normal cells become cancerous when they switch glucose metabolism from oxidative phosphorylation to aerobic glycolysis [12].

Patients in whom the Warburg effect is manifested have a very poor prognosis. It has been reported that the mortality rate of lactic acidosis in patients with cancer exceeds $80 \%$ [5]. Previous reports have shown that only 2 of 28 patients with non-Hodgkin lymphoma with the Warburg effect achieved complete remission and that more than $75 \%$ of the patients died within a month [6].

There are several reports of lactic acidosis associated with DLBCL [13-15]. In one case report of a 54-year-old man diagnosed with DLBCL and lactic acidosis, the patient's condition continued to deteriorate despite chemotherapy, resulting in palliative care [13]. In another case, a 55-year-old woman with newly diagnosed DLBCL achieved complete resolution of lactic acidosis with chemotherapy and renal replacement therapy. The patient was discharged, but a few months later, she succumbed to the primary disease [14]. Masood et al. [15] reported a case of DLBCL in which the lactic acidosis improved after vitamin $B_{1}$ administration and emphasized that it is necessary to pay attention to the presence or absence of vitamin $B_{1}$ deficiency.

In the present case, the patient could survive the critical events induced by the Warburg effect with prompt introduction of chemotherapy for DLBCL. Although the patient is still under treatment, this case indicates that even in cases of deteriorating general condition in patients with cancer due to the Warburg effect, identifying the cause of lactic acidosis early and introducing cancer treatment promptly may improve the patient's prognosis.

Lactic acidosis is a non-specific finding, and it may be difficult to determine its cause. If lactic acidosis and/or hypoglycemia occurs in patients with malignant tumors, we should always consider the possibility of the Warburg effect. This may be overlooked, as these changes may be considered as one of the terminal symptoms of cancer. The Warburg effect is a rare but serious condition that needs to be recognized, not only in DLBCL but in many malignancies. Early and appropriate cancer treatment may improve the quality of life and prognosis of patients with the Warburg effect with lactic acidosis and/or hypoglycemia, as in the present case.

\section{Acknowledgement}

We would like to thank the Clinical Research Support Team in Jichi Medical University for their guidance and cooperation in the preparation of this paper.

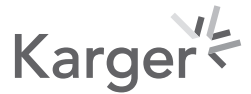




\section{Case Reports in Oncology}

Case Rep Oncol 2020;13:1047-1052

\begin{tabular}{l|c|}
\hline DOI: 10.1159/000509510 & $\odot 2020$ The Author(s). Published by S. Karger AG, Basel
\end{tabular} www.karger.com/cro

Hamada et al.: Lactic Acidosis and Hypoglycemia in Gastric DLBCL due to Warburg Effect

\section{Statement of Ethics}

The patient and her family provided written informed consent for the publication of her data and any related images.

\section{Conflict of Interest Statement}

The authors have no conflicts of interest to declare.

\section{Funding Sources}

No funding was received.

\section{Author Contributions}

T.H. conceived the need to describe the case and wrote the manuscript. T.H. and N.O. cared for this patient. H.Y. revised the manuscript for important intellectual content. Y.M. contributed to the concept and helped to write the manuscript. All other authors have critically reviewed the manuscript. All authors read and approved the final version of the manuscript.

\section{References}

1 Potter M, Newport E, Morten KJ.The Warburg effect: 80 years on. Biochem Soc Trans. 2016;44(5):1499-505

2 Siska PJ, Singer K, Evert K, Renner K, Kreutz M. The immunological Warburg effect: can a metabolic-tumorstroma score (MeTS) guide cancer immunotherapy? Immunol Rev. 2020;295(1):187-202.

3 De Raes EA, Benoit DD, Depuydt PO, Offner F, Nollet J, Vantilborgh AK, et al. Early recognition of malignant lactic acidosis in clinical practice: report on 6 patients with haematological malignancies. Acta Clin Belg. 2012; 67(5):347-51.

4 Andersen LW, Mackenhauer J, Roberts JC, Berg KM, Cocchi MN, Donnino MW. Etiology and therapeutic approach to elevated lactate levels. Mayo Clin Proc. 2013;88(10):1127-40.

5 Martinez-Outschoorn UE, Whitaker-Menezes D, Valsecchi M, Martinez-Cantarin MP, Dulau-Florea A, Gong J, et al. Reverse Warburg effect in a patient with aggressive B-cell lymphoma: is lactic acidosis a paraneoplastic syndrome? Semin Oncol. 2013;40(4):403-18

6 He YF, Wei W, Sun ZM, Ji CS, Wang G, Chen MP, et al. Fatal lactic acidosis and hypoglycemia in a patient with relapsed natural killer/T-cell lymphoma. Adv Ther 2007;24(3):505-9.

7 Fall PJ, Szerlip HM. Lactic acidosis: from sour milk to septic shock. J Intensive Care Med. 2005;20(5):255-71.

8 Dean RK, Subedi R, Gill D, Nat A. Consideration of alternative causes of lactic acidosis: thiamine deficiency in malignancy. Am J Emerg Med. 2017;35(8):1214-e6.

9 Kraut JA, Madias NE. Lactic Acidosis: Current Treatments and Future Directions. Am J Kidney Dis. 2014;68(3): 473-82.

10 de Groot R, Sprenger RA, Imholz AL, Gerding MN. Type B lactic acidosis in solid malignancies. Neth J Med. 2011; 69(3):120-3.

11 Liberti MV, Locasale JW. The Warburg effect: how does it benefit cancer cells? Trends Biochem Sci. 2016; 41(3):211-8.

12 Devic S. Warburg effect - a consequence or the cause of carcinogenesis? J Cancer. 2016;7(7):817-22.

13 Arif H, Zahid S, Kaura A. Persistent lactic acidosis: thinking outside the box. Cureus. 2018;10(5):e2561.

14 He Y, Ong J, Ong S. Refractory lactic acidosis and an approach to its management - a case report. J Crit Care Med. 2019;5(2):60-5.

15 Masood U, Sharma A, Nijjar S, Sitaraman K. B-cell lymphoma, thiamine deficiency, and lactic acidosis. Proc (Bayl Univ Med Cent). 2017;30(1):69-70. 